\title{
Osmotic controlled drug delivery system (OSMO technology) and its impact on diabetes care
}

\author{
Rajesh Rajput ${ }^{1 *}$, K. M. K. Prasanna Kumar², D. S. Arya ${ }^{3}$, A. K. Das ${ }^{4}$, A. H. Zargar ${ }^{5}$, Mangesh \\ Tiwaskar $^{6}$, Vijay Motghare ${ }^{7}$, Ruchi Shah ${ }^{8}$, Shahu Ingole ${ }^{8}$, Rishi Jain ${ }^{8}$
}

\author{
${ }^{1}$ Department of Endocrinology, PGIMS Rohtak, Haryana, India \\ ${ }^{2}$ Department of Endocrinology, Center for Diabetes and Endocrine Care, Bangalore Diabetes Hospital, Bangalore, \\ Karnataka, India \\ ${ }^{3}$ Department of Pharmacology, All India Institute of Medical Sciences, New Delhi, India \\ ${ }^{4}$ Department of Medicine and Endocrinology, Pondicherry Institute of Medical Sciences, Pondicherry, India \\ ${ }_{5}^{5}$ Department of Endocrinology, Centre for Diabetes and Endocrine Care, National Highway Gulshan Nagar, Srinagar, \\ Jammu and Kashmir, India \\ ${ }^{6}$ Department of Medicine and Endocrinology, Shilpa Medical Research Centre, Dahisar East, Mumbai, Maharashtra, \\ India \\ ${ }^{7}$ Department of Pharmacology, Government Medical College, Nagpur, Maharashtra, India \\ ${ }^{8}$ Department of Medical Affairs, Wockhardt Ltd, Mumbai, Maharashtra, India
}

Received: 14 October 2020

Accepted: 18 November 2020

\section{*Correspondence:}

Dr. Rajesh Rajput,

E-mail: drrajeshrajput@ outlook.com

Copyright: ( $)$ the author(s), publisher and licensee Medip Academy. This is an open-access article distributed under the terms of the Creative Commons Attribution Non-Commercial License, which permits unrestricted non-commercial use, distribution, and reproduction in any medium, provided the original work is properly cited.

\begin{abstract}
Recently, focus on the development of controlled release drug delivery system has increased, as existing drugs exhibit certain pharmacokinetic limitations. The major goal of designing sustained release formulations is to improve the drug performance by prolonged duration of drug action, decreased frequency of dosing and reduced side effects by using smallest quantity of drug administered by the most suitable route. Osmotic-controlled release oral delivery system (OSMO technology) is the most promising strategy based system for sustained delivery of drug. Drug can be delivered in a controlled manner over a long period of time by the process of osmosis. Osmotic drug delivery system appears to be a promising solution for the limitations of conventional extended release formulations by virtue of their distinguished technological features. The present review describes briefly about various controlled drug delivery systems with special focus on advantages of osmotic-controlled release oral delivery system related to diabetes therapy and improved compliance.
\end{abstract}

Keywords: Osmo technology, Controlled drug delivery, Sustained release, Metformin gastrointestinal intolerability, Adherence

\section{INTRODUCTION}

Oral route is the most commonly and widely utilized route of administration among all the routes for systemic delivery of drugs. The reasons for such widespread use of oral route are its ease of administration, better patient acceptance and cost-effective manufacturing process. As compared to parenteral route, it is regarded as the most natural, uncomplicated, convenient and safer route of drug administration. ${ }^{1}$ Lot of research is going on the development of controlled release drug delivery system as existing oral drug delivery systems exhibit certain pharmacokinetic limitations and moreover there is risk increased side effects due to multiple drug therapy in chronic diseases like diabetes. Delivering drug at controlled rate, slow delivery, targeted delivery are other 
very attractive methods and have been pursued vigorously. Though various technologies used in drug formulations to control the rate of delivery seem similar in achieving delayed drug delivery, there are technological differences which make each one these technologies different from each other. As these technological differences in controlled release drug delivery system result into different outcomes, it is necessary to understand the mechanism of these technologies and their benefits. The key objective of this comprehensive review is to highlight the key features of various technologies used for providing sustained release of drug with focus on osmotic controlled drug delivery system and impact it can have in clinical practice.

\section{CONVENTIONAL ODDS}

A drug delivery system (DDS) is defined as a formulation or a device that enables the introduction of a therapeutic substance into the body and improves its efficacy and safety by controlling the rate, time, and place of release of drugs in the body. Drugs for oral delivery are mainly categorised into immediate release or conventional drug delivery systems and controlled release systems. Conventional oral drug delivery systems are designed for immediate release of drug for rapid absorption. Bioavailability of drug from these formulations may vary significantly depending on factors like physicochemical properties, presence of excipients, physiological factors like presence of food, $\mathrm{pH}$, gastrointestinal (GI) motility, etc. $^{2}$

These immediate release dosage forms have some limitations such as: frequent administration, poor patient compliance, difficulty in attaining steady state plasma concentration-time profile, and increased side effects due to fluctuating drug levels. Thus, to overcome these disadvantages, controlled drug delivery systems have been developed. ${ }^{1}$

\section{CONTROLLED DRUG DELIVERY SYSTEMS}

Controlled drug delivery systems are capable of controlling the rate of drug delivery, sustaining the duration of therapeutic activity and/or targeting the delivery of drug to a tissue. Controlled drug delivery can be defined as: sustained drug action at a predetermined rate by maintaining a relatively constant, effective drug level in the body with concomitant minimization of undesirable side effects; targeted drug action by using carriers or chemical derivatives to deliver drug to a particular target cell type; and provide a physiologically/therapeutically based drug release system. ${ }^{1}$

Controlled drug delivery or modified drug delivery systems are divided into four categories: delayed release, sustained release, site-specific targeting, and receptor targeting. ${ }^{3-6}$

The main aim of developing sustained release (SR) formulations is to prolong duration of drug action, decrease the dose and frequency, reduce side effects and improve overall drug performance. SR dosage forms provide sustained drug release pattern to maintain therapeutic level for a certain predetermined time. ${ }^{?}$

Advantages of SR: less frequency of dosing, reduced fluctuation in steady-state drug levels, maximum utilisation of the drug, increased safety margin of potent drug, shorter treatment period, overall reduction in health care costs, improved patient compliance, and overall better treatment outcomes. ${ }^{7}$

Table 1: Classification of sustained release drug delivery systems.

\begin{tabular}{|c|c|}
\hline Classification & Types \\
\hline \multirow{5}{*}{ Diffusion systems } & Reservoir devices \\
\hline & Matrix devices \\
\hline & Hydrophobic matrix system \\
\hline & Hydrophilic matrix system \\
\hline & Fat-wax matrix system \\
\hline \multirow{3}{*}{ Dissolution sustained systems } & Soluble reservoir system \\
\hline & Soluble matrix system \\
\hline & Dissolution sustained pulsed delivery system \\
\hline \multicolumn{2}{|c|}{ Ion exchange resins sustained release systems } \\
\hline \multirow{8}{*}{ Methods using osmotic pressure } & Elementary osmotic pump (EOP) \\
\hline & Push pull osmotic pump \\
\hline & Osmotic pump with non-expanding second chamber \\
\hline & Controlled porosity osmotic pump \\
\hline & Osmotic bursting osmotic pump \\
\hline & OROS-CT \\
\hline & Multi particulate delayed release systems \\
\hline & Liquid oral osmotic system (L-OROS) \\
\hline Mineral matrices & Macro porous systems \\
\hline
\end{tabular}




\begin{tabular}{|l|l|}
\hline Classification & \multicolumn{1}{|c|}{ Types } \\
\cline { 2 - 2 } & Micro porous system \\
\hline pH Independent formulations & \\
\hline Altered density formulations & \\
\hline Swelling and expansion systems & \\
\hline Floating systems & \\
\hline Bio adhesive or mucoadhesive systems & \\
\hline Biodegradable matrices & \\
\hline
\end{tabular}

\section{OSMOTICALLY CONTROLLED DRUG DELIVERY SYSTEMS}

Osmotic drug delivery system, is the most promising strategy-based drug delivery system to enhance drug bioavailability. ${ }^{8}$

Osmotic systems are based on the principle of osmosis. Such systems release the drugs at a constant zero order rate. These systems are popularly known as osmoticcontrolled release oral delivery system (OROS). The system consist of a drug core and an osmotically active substance (osmogen) like mannitol surrounded by a semipermeable membrane coating with an orifice of 0.4 $\mathrm{mm}$ made by laser beam to facilitate drug exit. When exposed to gastrointestinal fluid, water flows though semipermeable membrane and under the influence of osmotic force of osmogen, the drug release is facilitated via orifice. ${ }^{9}$

\section{Classification of osmotic drug delivery systems}

Osmotic drug delivery devices fall in two categories: implantable and oral. ${ }^{10,11}$

\section{Implantable osmotic drug delivery devices}

Implantable pump is composed of three layers: innermost layer is of the drug reservoir which is surrounded by an osmotic sleeve having the osmotic agent and outermost layer contains the rate controlling semi permeable membrane. Water enters through semi permeable membrane and acts with the osmotic agent thereby displacing the drug through its reservoir. These pumps are available with variety of delivery rates between 0.25 to 10 $\mathrm{ml}$ per hour and delivery duration between one day and four weeks. ${ }^{11,12}$

\section{Oral osmotic drug delivery devices}

The most widely accepted osmotic drug delivery system is OROS. The most important feature is that this system is independent of the physiological factors of the gastrointestinal tract and can be utilized for systemic as well as targeted delivery of drugs. ${ }^{13-15}$

Oral osmotic pump are of various types: single chamber osmotic pump - elementary osmotic pump; multi chamber osmotic pump - push pull osmotic pump and osmotic pump with nonexpanding second chamber; and specific types of osmotic pumps i.e. controlled porosity osmotic pump, osmotic bursting osmotic pump, liquid OROS, delayed delivery osmotic device, telescopic capsule, OROS colon targeting (CT), sandwiched oral therapeutic system, monolithic osmotic systems, and osmotic matrix tablet (OSMAT).

From the original 'elementary' osmotic pump to the pushpull system and consequently the advanced longitudinally compressed tablet (LCT) multilayer formulation, OSMO technology has developed through the years. ${ }^{13}$ All these technologies utilize the semi-permeable membrane which controls the rate of drug delivery. ${ }^{16}$ There are some differences in these various types of osmotic pumps. Covering the details of each type is beyond the scope of this article and only two commonly used technologies i.e. push pull technology and monolithic system are described in detail.

\section{Multi chamber osmotic pump- push pull osmotic pump}

The push-pull osmotic tablet designed with a bilayer or trilayer core consists of a drug layer and excipients and a push layer containing a hydrophilic expanding compartment which are osmotically active, it resembles a standard bilayer coated tablet. ${ }^{13,16}$ This push layer generates a hydrodynamic pressure pushing the drug through single or multiple passage ways. ${ }^{16}$ Thus, upon ingestion, both layers of the tablet facilitate absorption of water from the GI tract, forming a suspension or a solution in the drug layer. Since the drug can be delivered via suspension, this system is capable of delivering drugs with varied solubility, including water-insoluble drugs. ${ }^{13}$ Figure describes about the different layers of the tablet, containing an over coat of the drug, then a semipermeable membrane, underneath which contains another drug layer over a layer of osmotically activated push layer (Figure 1). ${ }^{10}$

\section{Monolithic osmotic systems}

The main principle used in this system is dispersion of water-soluble agent in a polymer matrix. In the monolithic system, there is a reservoir on the interior consisting of mixture of drug, osmotic agent, gelling agent and wetting agent. This is surrounded by a polymer matrix layer. Absorption of water ruptures polymer matrix capsule thus liberating the drug to the exterior (Figure 2). ${ }^{10}$ 


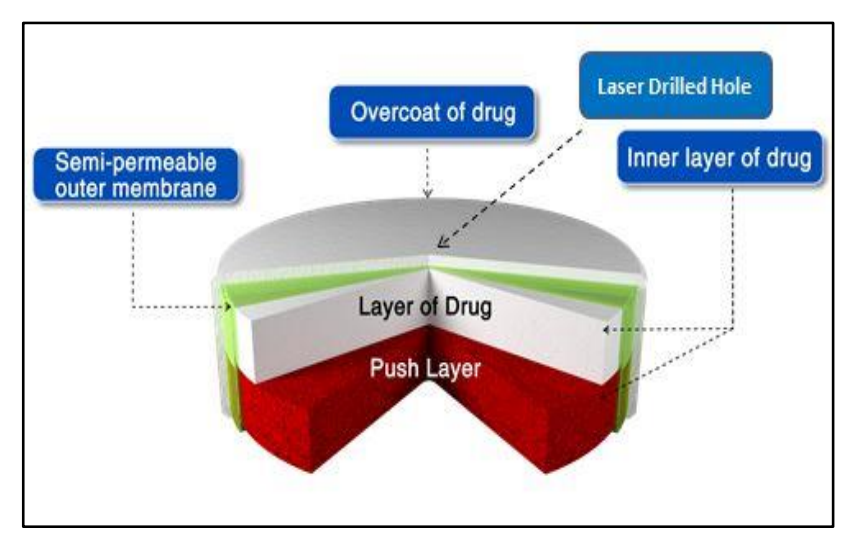

Figure 1: Push-pull osmotic tablet.

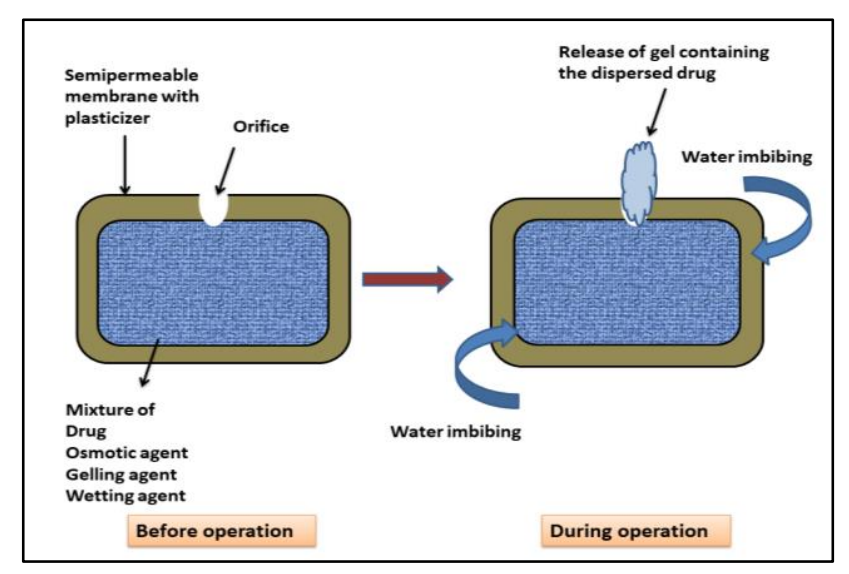

Figure 2: Monolithic osmotic system.

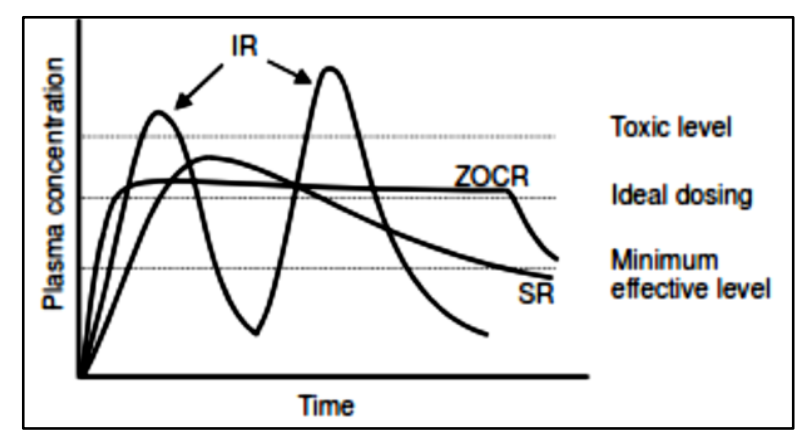

Figure 3: Graphical representation of plasma concentrations of a conventional immediate release (IR), a sustained release (SR) and an idealized zeroorder controlled release (ZOCR) drug delivery systems i.e. OROS.

\section{Advantages of osmotic drug delivery systems}

The following advantages have resulted in the popularity of osmotic drug delivery systems: zero-order drug delivery rate, delivery of drug may be delayed or pulsed, as per requirement, drug release is independent of gastric $\mathrm{pH}$ and hydrodynamic state, highly predictable drug release rate, and a high degree of in vivo-in vitro correlation (IVIVC). ${ }^{10,17}$

\section{MARKETED OROS PRODUCTS}

The products that have been developed and marketed based on osmotic drug delivery technology cover primarily the following four therapeutic areas: cardiovascular (35\%), neurological (25\%), seasonal (25\%) and metabolic disorders $(15 \%)$. In the cardiovascular therapeutic area, this system has been developed for two calcium channel blockers; nifedipine and verapamil; in the field of endocrinology, for sulfonylurea glipizide; in urology for doxazosin which is used for the treatment of the symptoms of benign prostatic hyperplasia (BPH), and oxybutynin for the treatment of overactive bladder; in the central nervous system (CNS) therapeutic area, the stimulant methylphenidate, combined with the advanced LCT multilayer delivery formulation, and a new psychotropic drug called paliperidone ER. ${ }^{13}$

\section{DIABETIC CARE: PROBLEM OF MEDICATION NON-ADHERENCE}

Plethora of oral antidiabetic medications are available today for diabetes management including metformin, sulfonylurea, dipeptidyl peptidase inhibitors-4 (DPP4) inhibitors, sodium-glucose co-transporter-2 (SGLT-2) inhibitors, Glucagon-like peptide (GLP) analogues, etc. However they are challenged by non-adherence. Medication non-adherence is multifactorial with various factors contributing such as age, medication information, perception and duration of disease, size of tablet and complexity of dosing regimen, polytherapy, inability to maintain/track health records and monitor treatment, psychological factors, safety, tolerability, comorbidities and cost. ${ }^{18}$ The World Health Organization (WHO) has shown that adherence to long-term therapy for chronic illnesses in developed countries averages only around $50 \% .{ }^{18}$ Ensuring patient compliance is one of the most challenging issues in chronic diseases like diabetes mellitus.

Diabetes mellitus (DM) is a complex, chronic, heterogeneous endocrine disorder wherein medication adherence is extremely crucial so as to achieve better therapeutic outcomes and the drug regimen is such that makes compliance challenging. ${ }^{19}$ Data suggests that $70 \%$ type 2 diabetes patients reported non-adherence to antidiabetic medications. ${ }^{20}$ In a study of 2,38,000 type 2 DM (T2DM) patients; adherence rate was found to be $47 \%$ with DPP4 inhibitors, $41 \%$ with sulphonylureas and $37 \%$ with thiazolidinediones. ${ }^{21}$ Non-adherence to anti diabetic medication is associated with inadequate glycemic control, increased morbidity and mortality and overall increased costs. These include costs of outpatient care, emergency room visits, hospitalization and managing complications of diabetes. ${ }^{20,22,23}$ Data suggests that at least $45 \%$ of patients with T2DM fail to achieve adequate glycemic control $(\mathrm{HbA} 1 \mathrm{c}<7 \%)$ and one of the major contributing factors is poor medication adherence. ${ }^{24}$ 
Frois and colleagues showed that serial non-adherence is sizable and is a key predictor of subsequent non-adherence which consequently results in incrementally higher medical care costs, elevated risk for hypoglycemia and poor patient outcomes compared with medication adherence. $^{25}$ A longitudinal 4 year study with 740,195 T2DM patients found that nonadherent patients can have annual inpatient costs $41 \%$ higher compared to adherent patients. ${ }^{26} \mathrm{~A}$ study with $15984 \mathrm{~T} 2 \mathrm{DM}$ patients reported that treatment noncompliance was associated with a significant $(\mathrm{p}<0.001) 1.6$ fold increase in all-cause mortality. ${ }^{27}$

There exists an inverse relationship between OHA adherence and $\mathrm{HbA} 1 \mathrm{c}$ level. The statistics reveal that each $10 \%$ increase in OHA adherence is associated with a decrease of $0.1 \%$ in HbA1c level. This indicates that level of adherence is a significant predictor of HbA1c, implying that improved adherence results in better glycemic control. ${ }^{18}$

\section{CAN OSMO TECHNOLOGY INCREASE MEDICATION ADHERENCE IN PATIENTS WITH TYPE 2 DIABETES MELLITUS?}

Most of the oral anti-diabetic drugs are available either in the immediate or extended release forms and these dosage forms result in increased probability of side effects, and complex dosage regimens which results into poor compliance. ${ }^{8}$

Sulfonylureas are among the first-line treatments because of their long history of proven efficacy and safety. The longer-acting and the short-acting sulfonylureas differ in their pharmacokinetic and pharmacodynamic profiles and, therefore, produce different therapeutic and side-effect profiles. Shorter-acting sulfonylureas, such as IR glipizide, are found to be more effective in improving mealstimulated insulin secretion and generally have a lower risk of hypoglycemia, but twice daily dosing (half-life 3-7 hours) is needed. Glipizide was designed as an ER preparation using the OROS technology to deliver oncedaily administration. Maximum plasma drug concentrations are attained by approximately 9 hours post dosing with OROS glipizide. With subsequent once-daily dosing, effective plasma concentrations are maintained throughout the 24 hour dosing interval with less peak-totrough fluctuation as opposed to the IR formulation. Furthermore, the once-daily regimen has proven to improve adherence and persistence to therapy compared to the twice-daily dosing of the IR formulation. ${ }^{13}$ Many other antidiabetic drugs like metformin, glibenclamide, gliclazide, glimepiride, pioglitazone, some GLP-1RA are available as extended release formulations. ${ }^{28}$

\section{METFORMIN GI INTOLERABILITY AND OSMO SOLUTION}

Metformin is the first line agent for T2DM patients but it is associated with gastrointestinal effects, including abdominal discomfort, nausea, and diarrhea, although these can be minimized by slow titration and administration with food. ${ }^{29}$ In a study conducted by Blonde et al percentage of patients with individual GI adverse events with metformin-XR and immediate-release metformin, respectively, were as follows: diarrhea $(6.77$ versus $7.59 \%$ ), nausea (2.26 versus $3.80 \%)$, dyspepsia (1.61 versus $1.27 \%$ ), abdominal pain (1.61 versus $0.63 \%)$, constipation $(0.97$ versus $0.63 \%)$, vomiting $(0.65$ versus $0.63 \%)$, abdominal distension (0.32 versus $0 \%)$, fecal abnormality $(0.32$ versus $0.63 \%)$, blood in stool ( 0 versus $0.63 \%)$, and flatulence ( 0 versus $0.63 \%) .{ }^{30}$ This indicates that still many patients suffer from intolerable GI side effects which are not addressed by currently available extended release formulations. Also, many patients who would otherwise benefit from metformin up-titration therapy discontinue treatment because of gastrointestinal symptoms, typically during the first few weeks of treatment. ${ }^{29}$ Extended release formulations offer various benefits to the patients thereby enhancing patient compliance. However, all these formulations are not similar because of different technology used. These technological differences in extended release formulations may impact patient compliance and safety. Therefore, a differentiated sustained release technology i.e. osmotic push pull technology which differs from many available technologies used for manufacturing of extended release formulations offers distinct clinical advantages to the patients with T2DM. Push pull technology ensures predictable therapeutic plasma concentrations thereby minimizing the risk of GI side effects. The lesser incidence of GI side effects with an osmotic drug delivery system will have a beneficial role to improve patient adherence to metformin therapy. This will help patients with T2DM to achieve their glycemic goals and eventually translate into better patient outcomes.

\section{BETTER PHARMACOKINETICS AND PHARMACODYNAMICS USING OSMO TECHNOLOGY}

Vildagliptin shows promising properties amongst the DPP-4 inhibitors with established safety and efficacy in patients with T2DM. Dose of vildagliptin needs to be halved to $50 \mathrm{mg}$ once daily in patients who are on SU or have moderate to severe renal impairment or ESRD. Considering higher prevalence of chronic kidney disease (CKD) in diabetes and the fact most patients uncontrolled on SU need addition of DPP4 inhibitor for better glycemic control, large number of patients need to be started on vildagliptin $50 \mathrm{mg}$ once daily instead of twice daily. This may deprive from achieving prolonged glycemic control in these patients. Pharmacokinetic studies have shown that vildagliptin $50 \mathrm{mg}$ in sustained-release dosage form through OSMO technology provide gradual release of the drug and thereby can achieve prolonged DPP-4 inhibition without compromising safety in these patients. Vildagliptin SR tablet $50 \mathrm{mg}$ showed above $80 \%$ DPP-IV inhibition for 20 hours and vildagliptin IR tablet $50 \mathrm{mg}$ reported up to 13 hours. Weighted average percentage 
DPP-IV inhibition (0-18) and (0-24) was 91.0, 87.4 with vildagliptin $\mathrm{SR}$ and 85.1, 72.5 with vildagliptin IR, respectively. Drug was found to be safe and well tolerated. $^{31}$

Another study with metformin PR OSMO tablet versus metformin MR tablet showed that there is prolonged therapeutic action upto 24 hours, smoother uptitration and $30 \%$ lower Cmax thus less gastrointestinal intolerability with metformin PR OSMO tablet. ${ }^{32}$

\section{CONCLUSION}

Better understanding of pharmacodynamics and pharmacokinetics has demonstrated the need for newer drug delivery systems so as to achieve a constant, pulsatile, controlled, and triggered drug delivery. Osmotic drug delivery system is one such novel system that helps to deliver drugs in a controlled manner, provides predictable plasma concentration resulting into lesser side effects, and provides prolonged duration of action thereby improving the medication adherence in various disease conditions.

The clinical benefits have opened new perspectives for the future developments of newer oral delivery drugs. Osmotic drug delivery system appears to provide promising clinical benefits with antidiabetic medications by virtue of sustained therapeutic action and minimizing the side effects. Translation of these pharmacokinetics and pharmacodynamics benefits of osmotic drug delivery system into better clinical outcomes in practice needs to be evaluated in robust randomized controlled clinical trials.

Funding: No funding sources

Conflict of interest: None declared

Ethical approval: Not required

\section{REFERENCES}

1. Deepika B, Sameen S, Nazneen N, Madhavi A, Kandukoori NR, Dutt KR. Matrix drug delivery system-a review. Eur J Pharm Med Res. 2018;5(2):150-4.

2. Patel H, Patel U, Kadikar H, Bhimani B, Daslaniya D, Patel G. A Review On Osmotic Drug Delivery System. IRJP. 2012;3(4):88-94.

3. Jain KK. Drug delivery systems. 1st edition. Switzerland: Humana Press. 2008;1-51.

4. Paul B, Gupta PK, Ara HD, John EH. Remington the science and practice of pharmacy. 21st edition. New York: Wolter kluwer, Lippincott wiliams and Wilkins. 2006;939-62.

5. Edith M. Encyclopedia of controlled drug delivery. 1st edition. New York: A wiley interscience publication. 2009;381-5.

6. Gilbert SB, Christopher TR. Modern pharmaceutics. 3rd edition revised and expanded. New York: Marcel Dekker Inc. 1995;575-608.

7. Sahilhusen IJ, Mukesh RP, Alpesh DP. Sustained release drug delivery systems: a patent overview.
Aperito J Drug Designing and Pharmacology. 2014;1(1):1-4.

8. Rai VK, Mishra N, Agrawal AK, Jain S, Yadav NP. Novel drug delivery system: an immense hope for diabetics. Drug delivery. 2016;23(7):2371-90.

9. Ghosh T, Ghosh A. Drug delivery through osmotic systems - an overview. J Appl Pharm Sci. 2011;1(2):38-49.

10. Khatri N, Nikam S, Bilandi A. IJPSR. 2016;7(6):2302-12.

11. Pandey S, Devmurari V. Osmotic pump drug delivery devices: from implant to sandwiched oral therapeutic system. Int $\mathbf{J}$ Pharm Technol Res. 2010;2:693-9.

12. Mathur M, Mishra R. A review on osmotic pump drug delivery system. Int $\mathbf{J}$ Phar Sci and Res. 2016;1(7):453-67.

13. Conley R, Gupta SK, Sathyan G. Clinical spectrum of the osmotic-controlled release oral delivery system (OROS), an advanced oral delivery form. Curr Med Res Opinion. 2006;22(10):1879-92.

14. Gupta BP, Thakur N, Jain NP, Banweer J, Jain S. Osmotically controlled drug delivery system with associated drugs. J Pharm Pharmaceutic Sci. 2010;13(4):571-88.

15. Keraliya RA, Patel C, Patel P, Keraliya V, Soni TG, Patel RC, Patel MM. Osmotic drug delivery system as a part of modified release dosage form. ISRN pharmaceutics. 2012;1-9.

16. Malaterre V, Ogorka J, Loggia N, Gurny R. Oral osmotically driven systems: 30 years of development and clinical use. Eur J Pharm Biopharm. 2009;73(3):311-23.

17. Sowjanya M, Rao VP, Babu PS, Pallavi K. Osmotic Drug Delivery Systems: A Review. Inventi Rapid: NDDS. 2017;3:1-9.

18. Garcia-Perez LE, Alvarez M, Dilla T, Gil-Guillen V, Orozco-Beltran D. Adherence to therapies in patients with type 2 diabetes. Diabet Ther. 2013;4(2):175-94.

19. Sankar UV, Lipska K, Mini GK, Sarma PS, Thankappan KR. The adherence to medications in diabetic patients in rural Kerala, India. Asia Pacific J Public Health. 2015;27(2):513-23.

20. Sontakke S, Jadhav M, Pimpalkhute S, Jaiswal K, Bajait C. Evaluation of adherence to therapy in patients of type 2 diabetes mellitus. J Young Pharm. 2015;7(4):462.

21. Farr AM, Sheehan JJ, Curkendall SM, Smith DM, Johnston SS, Kalsekar I. Retrospective analysis of long-term adherence to and persistence with DPP-4 inhibitors in US adults with type 2 diabetes mellitus. Advances in Ther. 2014;31(12):1287-305.

22. Curkendall SM, Thomas N, Bell KF, Juneau PL, Weiss AJ. Predictors of medication adherence in patients with type 2 diabetes mellitus. Curr Med Res Opinion. 2013;29(10):1275-86.

23. Ahmad NS, Ramli A, Islahudin F, Paraidathathu T. Medication adherence in patients with type 2 diabetes mellitus treated at primary health clinics in Malaysia. Patient preference and adherence. 2013;7:525-30. 
24. Polonsky WH, Henry RR. Poor medication adherence in type 2 diabetes: recognizing the scope of the problem and its key contributors. Patient preference and adherence. 2016;10:1299-307.

25. Serial Medication Nonadherence in Patients with Type 2 Diabetes. Am Health Drug Benefits. 2015;8(2):S12-S16.

26. Luga AO, McGuire MJ. Adherence and health care costs. Risk management and healthcare policy. 2014;7:35.

27. Currie CJ, Peyrot M, Morgan CL, Poole CD, JenkinsJones S, Rubin RR, Burton CM, Evans M. The impact of treatment noncompliance on mortality in people with type 2 diabetes. Diabetes Care. 2012;35(6):1279-84.

28. Romera I, Cebrián-Cuenca A, Álvarez-Guisasola F, Gomez-Peralta F, Reviriego J. A review of practical issues on the use of glucagon-like peptide-1 receptor agonists for the management of type 2 diabetes. Diabet Ther. 2019;10(1):5-19.

29. Schwartz S, Fonseca V, Berner B, Cramer M, Chiang YK, Lewin A. Efficacy, tolerability, and safety of a novel once-daily extended-release metformin in patients with type 2 diabetes. Diabet Care. 2006;29(4):759-64.

30. Blonde L, Dailey GE, Jabbour SA, Reasner CA, Mills DJ. Gastrointestinal tolerability of extendedrelease metformin tablets compared to immediaterelease metformin tablets: results of a retrospective cohort study. Curr Med Res Opinion. 2004;20(4):565-72.

31. Data on file (Executive Summary for Vildagliptin SR tablets $50 \mathrm{mg}$ )

32. Data on file (PK-PD data for Metformin OSMO tablet $1000 \mathrm{mg})$.

Cite this article as: Rajput R, Kumar KMKP, Arya DS, Das AK, Zargar AH, Tiwaskar M, et al. Osmotic controlled drug delivery system (OSMO technology) and its impact on diabetes care. Int J Res Med Sci 2021;9:303-9. 